\title{
Relevance of initial and final conditions for the Fluctuation Relation in Markov processes.
}

\author{
A Puglisi ${ }^{1}$, L Rondoni $^{2}$ and A Vulpiani ${ }^{3}$ \\ ${ }^{1}$ Dipartimento di Fisica, Università La Sapienza, p.le Aldo Moro 2, 00185 Roma, \\ Italy \\ 2 Dipartimento di Matematica and INFM, Politecnico di Torino, Corso Duca degli \\ Abruzzi 24, I-10129, Torino, Italy \\ ${ }^{3}$ Dipartimento di Fisica and INFN, Università La Sapienza, p.le Aldo Moro 2, 00185 \\ Roma, Italy \\ E-mail: andrea.puglisi@roma1.infn.it
}

\begin{abstract}
Numerical observations on a Markov chain and on the continuous Markov process performed by a granular tracer show that the "usual" fluctuation relation for a given observable is not verified for finite (but arbitrarily large) times. This suggests that some terms which are usually expected to be negligible, i.e. "border terms" dependent only on initial and final states, in fact cannot be neglected. Furthermore, the Markov chain and the granular tracer behave in a quite similar fashion.
\end{abstract}




\section{Introduction}

Non-equilibrium systems are at the center of old but still very lively research in physics, due to the great importance that out of equilibrium phenomena have in the natural world 1, 2, 3. Since the seminal studies by Carnot, Clausius and Kelvin, who made clear the relevance of the lack of equilibrium in the extraction of work from heat, systems out of thermodynamic equilibrium are investigated in the vastest set of applications, ranging from mechanical engineering to the study of chemical reactions, molecular ratchets and all the biophysical processes of growth, differentiation, movement and evolution.

On the theoretical side, the study of fluctuations in non-equilibrium systems has been marked by a series of fundamental breakthroughs, such as Einstein's work 44 on Brownian motion and the first statement of a Fluctuation-Response theory, through its celebrated relation between diffusivity and mobility; the Onsager's works on the reciprocity of transport coefficients and on the regression hypothesis of large fluctuations [5]; and the works of Green and Kubo on the general linear response formalism and the Fluctuation-Dissipation relation (FDR) in its full dynamical form [6, 7]. All these works have resulted in the construction of the so-called NonEquilibrium Thermodynamics [8], that studies non-equilibrium states of smoothly varying locally equilibrated regions, i.e. of systems in local thermodynamic equilibrium.

The FDR links the mean response to a perturbation with a suitable correlation function computed for the unperturbed system, and was originally developed in the context of (equilibrium) statistical mechanics of Hamiltonian systems. This fact was misunderstood by some authors. For instance, it was claimed (with qualitative arguments) that in fully developed turbulence (which is a non Hamiltonian and non equilibrium system) there is no relation between fluctuations and relaxation to the statistical steady state [9]. In reality, a generalized FDR holds under rather general hypothesis, independently from the Hamiltonian nature of the systems [10, 11, 12]. If the system is mixing and the invariant measure is "smooth" enough, there exists a connection between the "non equilibrium" properties (response to external perturbations) and the "equilibrium" properties (correlation functions computed according to the equilibrium measure of the unperturbed system). The validity of the FDR does not depend on the deterministic or stochastic nature of the system neither on the "equilibrium" or "non equilibrium" character of its statistical steady state.

In the last decade a series of important results have been obtained, all related to a seemingly very general Fluctuation Relation (FR), that constrains without any fitting parameter the pdf of the fluctuations of entropy production or, more generally, of dissipated energy. The main steps, in historical order, are: the proposal and the observation of FR in numerical simulations, by Evans, Cohen and Morris in 1993 [13], a derivation of the same relation in transient states in 1994, by Evans and Searles [14, and the derivation of a theorem for asymptotic states by Gallavotti and Cohen in 1995 [15].

If the chaotic hypothesis of the Gallavotti-Cohen theorem holds, the deterministic dynamics can be mapped onto a mixing Markovian stochastic process: this observation 
stimulated the derivation of a stochastic version of the FR, first obtained by Lebowitz and Spohn [16] and, more rigorously (and under more restrictive hypothesis) by Maes [17. The case of Langevin equations has been discussed by Kurchan [18].

In this paper we will work in the stochastic framework, where one can directly speak of the probability, $\mathcal{P}_{\tau}(\Omega)$ say, of observing a given trajectory segment $\Omega$ of temporal length $\tau$. To begin with, we restrict our discussion to the simpler case of discrete states and, therefore, discrete trajectories. Then, in the presence of an appropriate form of reversibility, for each trajectory $\Omega$ one can define the quantity

$$
W^{\prime}(\Omega)=\log \frac{\mathcal{P}_{\tau}(\Omega)}{\mathcal{P}_{\tau}(\bar{\Omega})}
$$

where $\bar{\Omega}$ is the reversal of $\Omega \ddagger$. When detailed balance is satisfied one has $W^{\prime}(\Omega) \equiv 0$. In the following we will refer to such a case as to "equilibrium". Equation (1D) implies $\mathcal{P}_{\tau}(\Omega)=\exp \left(W^{\prime}(\Omega)\right) \mathcal{P}_{\tau}(\bar{\Omega})$ and, straightforwardly,

$$
\begin{aligned}
& \operatorname{prob}\left(W^{\prime}=X\right)=\sum_{\Omega: W^{\prime}(\Omega)=X} \mathcal{P}_{\tau}(\Omega)=\sum_{\Omega: W^{\prime}(\Omega)=X} \exp \left(W^{\prime}(\Omega)\right) \mathcal{P}_{\tau}(\bar{\Omega})= \\
& \exp (X) \sum_{\Omega: W^{\prime}(\Omega)=-X} \mathcal{P}_{\tau}(\Omega) \quad=\exp (X) \operatorname{prob}\left(W^{\prime}=-X\right) .
\end{aligned}
$$

The symmetry $\operatorname{prob}\left(W^{\prime}=X\right)=\exp (X) \operatorname{prob}\left(W^{\prime}=-X\right)$ is the simplest form of a $\mathrm{FR}$. This relation is the basis of a variety of FR's discussed in the recent literature [19].

In the treatment of Gallavotti and Cohen for deterministic systems, the time $\tau$ must be large, the role of $W^{\prime}$ is played by the time-integrated phase space contraction rate and the following large deviation function of $W^{\prime}$ is considered:

$$
\pi(x)=\lim _{\tau \rightarrow \infty} \frac{1}{\tau} \log \left(\operatorname { p r o b } \left(W^{\prime} \in((x-\delta) \tau,(x+\delta) \tau), \quad \text { for any } \delta>0 .\right.\right.
$$

The FR for $W^{\prime}$ can then be written as

$$
x-\delta \leq \pi(x)-\pi(-x) \leq x+\delta .
$$

The idea underlying definition (11) is to identify heuristically the contribution of a single trajectory to the entropy production, so that $W^{\prime}$ physically represents an integrated flux (e.g. heat flux) divided by a temperature (or a sum of fluxes divided by the temperatures of the different reservoirs involved), i.e. $W^{\prime}=\beta E Y$ where $E$ is the applied non-conservative field, $Y$ is its conjugated observable and $E \times Y$ is an energy. For small Gaussian fluctuations, the FR yields directly $\left\langle W^{\prime 2}\right\rangle_{c} \equiv\left\langle W^{\prime 2}\right\rangle-\left\langle W^{\prime}\right\rangle^{2}=2\left\langle W^{\prime}\right\rangle$, because of the parabolic shape of the large deviation function. Then the classical FDR emerges as a particular case near equilibrium (i.e. near $\left\langle W^{\prime}\right\rangle=0$ ). In fact, the previous physical identification of $W^{\prime}$ implies $\beta\left\langle Y^{2}\right\rangle_{c}=2\langle Y\rangle / E$. Therefore, assuming that $\left\langle Y^{2}\right\rangle_{c}=2 D t$ where $D$ is given by its equilibrium value (as it is true to first order in $E$ ) and $\langle Y\rangle=m E t$ (linear response), one obtains $m=\beta D$ which is the celebrated Einstein relation between mobility $m$ and diffusivity $D$. In a similarly straightforward

$\ddagger$ In section II the quantity denoted by $W^{\prime}$ will be contrasted with a different quantity denoted simply by $W$. 
fashion, the Onsager reciprocal relations can be derived from the FR, recalling the time reversibility of the evolution.

In deterministic dynamics, the Gallavotti-Cohen theorem is based on the hypothesis that the system is Anosov, hence that its phase space is bounded. In some systems (for example in presence of singular potentials) this hypothesis is not well controlled, because non-conservative forces let $W^{\prime}$ fluctuate without bounds. In the stochastic framework, this problem can be cast into simple mathematical terms. In particular, Ref. [16] considers a Markov chain, in the stationary state, with probability of each trajectory $\Omega$ given by

$$
\mathcal{P}(\Omega)=\mu_{\sigma_{0}} \Pi_{i=0}^{\tau-1} K_{\sigma_{i} \sigma_{i+1}}
$$

where $\mu_{\sigma_{0}}$ is the invariant probability of the state $\sigma$ and $K_{\sigma_{i} \sigma_{i+1}}$ is the transition probability associated with the jump $\sigma_{i} \rightarrow \sigma_{i+1}$ between two possible states of the chain. Therefore, the functional $W^{\prime}$ can be divided in two pieces:

$$
W^{\prime}=W+B
$$

where $B$ is $\log \mu_{\sigma_{0}} / \mu_{\sigma_{\tau}}$. In this paper we will argue that the fluctuations of $B$, which has to be considered as a "border" term, are of practical importance (i.e. in numerical and, presumably, also in real experiments) in the measurements of $W$. The relevance of border terms for finite and infinite times has been discussed recently in other contexts: Farago 20] was the first, to the best of our knowledge, to show with exact results the difference of the large deviations of work done by the thermostat and energy dissipated by the system, in stochastic models described by a Langevin equation. Border terms had been dealt with also in deterministic dynamics, in the paper [21] by Evans and Searles, where the "dissipation function" $f$, analogous to our $W^{\prime}$, was introduced as a physically relevant quantity. In a time dependent Langevin equation, van Zon and Cohen [22] and more recently Baiesi and co-workers [23] have studied the difference between work done and heat transferred to the thermostat; the effect of a border term making the difference between two heat fluxes in a non-equilibrium Langevin model has been studied with exact analytical results in a more recent paper by Visco [24]. Simulations of deterministic system [25] have revealed the importance of border terms also at finite times. In Ref. 26] the necessity of the inclusion of border terms was recognized, and the example of a Bernoulli process has been discussed. In the Lebowitz-Spohn formulation, the problem of borders at finite times has been treated in [27], considering simulations of a tracer particle in a granular gas. The necessity of including border terms has also been recognized in 28 .

The term $B$ can contribute to the large deviations of $W$, even if all time-rescaled cumulants of its fluctutations vanish. Van Zon and Cohen 22] first made a general observation: if one has a time-extensive variable $Y(t)$ (an observable whose cumulants all grow linearly in time $\S$ ) and a non extensive variable $X$ with unbounded fluctuations

$\S$ We informally use the term extensive, referring to the "time-Gibbsian property" (see for example [17) of the measure of $\mathcal{P}(\Omega)$ and therefore of $\operatorname{prob}(W=X)$ : this amounts to say that the probability of 
whose probability has exponential tails, or slower, then there is no guarantee that the extensive variable $Z(t)=X+Y(t)$ has the same large deviations of $Y(t)$. Heuristically, this observation may descend from the existence and finiteness of the large deviation function for $X$,

$$
\pi(x)=\lim _{t \rightarrow \infty} \frac{1}{t} \log \operatorname{prob}(X \in((x-\delta) t,(x+\delta) t) .
$$

On practical grounds, we will show that the "memory" of initial and final conditions, i.e. the relevance of $B$, lasts much longer $\left(10^{3}\right.$ times longer and more) than the typical time defined in terms of the probability evolution of the Markov process toward the invariant probability, making the $\mathrm{FR}$ for $W$ practically impossible to verify in almost all our examples. Recent theoretical studies [23] predict that the form of the violation of the FR for $\mathrm{W}$ should have given character in general, so that the resulting $\mathrm{FR}$ would take the form of the heat FR of Van Zon and Cohen. These studies concern a given class of systems and observables, hence it is interesting to see which other systems verify the same predictions and which systems do not. For instance, Ref. [29] shows models in which the violation of the FR takes on a different form. Therefore, we investigate also this question.

In section II we will pose the problem in a general form. Our two stochastic examples, a Markov chain with a large number of states and a continuous time Markov process exactly defined by the dynamics of a tracer in a granular gas [27, will be discussed respectively in Section III and IV. Concluding remarks will be given in section $\mathrm{V}$.

\section{Action functionals in Markov chains}

\subsection{Definitions and properties}

Consider a Markov chain with $N$ (possibily infinite) states with invariant probability measure $\boldsymbol{\mu}$ and transition rates $K_{a b}$ which denote the conditional probability of going from state $a$ to state $b$, we are interested in the fluctuations of the following two "action functionals":

$$
\begin{aligned}
W(\tau) & =\sum_{i=0}^{\tau} \log \frac{K_{\sigma_{i} \sigma_{i+1}}}{K_{\sigma_{i+1} \sigma_{i}}} \\
W^{\prime}(\tau) & =\sum_{i=0}^{\tau} \log \frac{K_{\sigma_{i} \sigma_{i+1}}}{K_{\sigma_{i+1} \sigma_{i}}}+\log \frac{\mu_{\sigma_{0}}}{\mu_{\sigma_{\tau+1}}}
\end{aligned}
$$

where $\sigma_{i} \in\{1,2, \ldots N\}$ is the state of the system at time $i$. Both functionals have been defined by Lebowitz and Spohn, in [16], who focused on the properties of $W$, neglecting the importance of the difference $B=W^{\prime}-W$. For this reason, we call $W$ as the "Lebowitz-Spohn functional", while we refer to $W^{\prime}$ as to the "corrected" or

$W$ obeys a large deviation scaling, being time the "large parameter", or in other words that it is characterized by an exponential decay when the time length of the trajectory is linearly increased. 
"adjusted" functional. Both $W$ and $W^{\prime}$ associate a real number to any finite trajectory (any realization of the Markov chain), and have the following properties:

- Zero at Equilibrium: by equilibrium we mean a stationary state which verifies the detailed balance condition $\mu_{a} K_{a b}=\mu_{b} K_{b a}$, being $\boldsymbol{\mu}=\left\{\mu_{i}\right\}$ the invariant measure. Then, one has $W(t) \equiv 0$ if $K_{a b}=K_{b a}$, even during transient states represented by measures other than the microcanical one. However, $W(t) \neq 0$ even at equilibrium except for isolated (microcanonical) systems. Differently, $W^{\prime}(t) \equiv 0$ at any equilibrium state, but not in transient states.

- Ergodicity: for $t$ large enough, for almost all the trajectories $\lim _{s \rightarrow \infty} W(s) / s=$ $\lim _{s \rightarrow \infty} W^{\prime}(s) / s=\langle W(t) / t\rangle=\left\langle W^{\prime}(t) / t\right\rangle$; here (assuming an ergodic and stationary system) \langle\rangle indicates an average over many independent segments from a single very long trajectory.

- Entropy production: Let $S(t)=-\sum_{i=1}^{N} \nu_{i} \log \nu_{i}$ be the entropy of the system at time $t$, where $\nu_{i}(t)$ is the probability to be in the state $i$ at time $t$; then

$$
S(t+1)-S(t)=R(t)-A(t)
$$

where $R(t)$ is always non-negative, $A(t)$ is a linear function with respect to $\boldsymbol{\nu}(t)=\left\{\nu_{i}(t)\right\}$, and $\langle W(t)\rangle=\left\langle W^{\prime}(t)\right\rangle \equiv \int_{0}^{t} \mathrm{~d} t^{\prime} A\left(t^{\prime}\right)$. In [16] this has been shown for continuous time Markov processes, but the proof is valid also in the discrete time case (see for example [30]). This leads to consider $W(t)$ and $W^{\prime}(t)$ equivalent to the contribution of a single trajectory to the total entropy flux. In a stationary state $A(t)=R(t)$ and therefore the flux is equivalent to the production.

- Positivity of the average: for large enough $t$ : 1 ) at equilibrium (i.e. when there is detailed balance) $\langle W(t)\rangle=\left\langle W^{\prime}(t)\right\rangle=0 ; 2$ ) out of equilibrium those two averages are positive.

Let us now introduce our objects of study:

- $F R_{W}: \pi(w)-\pi(-w)=w$ where $\pi(w)=\lim _{t \rightarrow \infty} \frac{1}{t} \log f(t, t w)$ and $f(t, x)$ is the probability of finding $W(t)=x$ at time $t$. Note that, in principle $\Pi(t, w)=$ $\frac{1}{t} \log f(t, t w) \neq \pi(w)$ at any finite time; a derivation of this property has been obtained in [16], while a rigorous proof with more restrictive hypothesis is in [17]; the discussion for the case of a Langevin equation is in [18].

- $F R_{W^{\prime}}: \Pi^{\prime}(t, w)-\Pi^{\prime}(t,-w)=w$ where $\Pi^{\prime}(t, w)=\frac{1}{t} \log f^{\prime}(t, t w)$ and $f^{\prime}(t, x)$ is the probability density function of finding $W^{\prime}(t)=x$ at time $t$.

\subsection{Fluctuations of $W$}

We first define an extended probability vector $\mathbf{p}(t, W)$ where each component $p_{i}(t, W)$ is the probability of finding the system at time $t$ in the state $i$ with the value $W$ for the Lebowitz and Spohn functional. This means that $\sum_{i} p_{i}(t, W)=f(t, W)$ is the probability density function at time $t$ for the functional $W$, while $\int \mathrm{d} W p_{i}(t, W)=\nu_{i}(t)$ 
is the probability of finding the system in state $i$ at time $\mathrm{t}$ and $\int \mathrm{d} W \sum_{i} p_{i}(t, W)=1$. We will use the simple notation $\mu_{i}$ to denote the invariant measure, i.e. $\mu_{i}=\lim _{t \rightarrow \infty} \nu_{i}(t)$. The evolution of $\mathbf{p}(t, W)$ is given by the following equation:

$$
p_{i}(t+1, W)=\sum_{j} K_{j i} p_{j}\left(t, W-\Delta w_{j i}\right)
$$

where $K$ is the previously defined transition matrix and $\Delta w_{i j}$ is the variation of $W$ due to a jump from the state $i$ to the state $j$. This reads $\Delta w_{i j}=\ln \frac{K_{i j}}{K_{j i}}$ for the original Lebowitz-Spohn functional.

Then define the function $\tilde{\mathbf{p}}(t, \lambda)=\int \mathrm{d} W \exp (-\lambda W) \mathbf{p}(t, W)$ and obtain, for its evolution

$$
\tilde{p}_{i}(t+1, \lambda)=\sum_{j} K_{j i} \tilde{p}_{j}(t, \lambda) e^{-\lambda \Delta w_{j i}}
$$

In a compact form we can write

$$
\tilde{\mathbf{p}}(t+1, \lambda)=A(\lambda) \tilde{\mathbf{p}}(t, \lambda)
$$

$A(\lambda)$ being the matrix defined by

$$
A_{i j}(\lambda)=K_{j i}^{1-\lambda} K_{i j}^{\lambda},
$$

and, therefore,

$$
\tilde{\mathbf{p}}(t, \lambda)=A(\lambda)^{t} \tilde{\mathbf{p}}(0, \lambda)
$$

with $\tilde{p}_{i}(0, \lambda)=\int \mathrm{d} W \exp (-\lambda W) \nu_{i}(0) \delta(W)=\nu_{i}(0)$.

Note that the $i j$-term of the evolution matrix $\tilde{f}_{i j}(t, \lambda)=\left[A(\lambda)^{t}\right]_{i j}$ is a function of $\lambda$ that represents the characteristic function of the distribution of $W$ at time $t$ constrained by the conditions $\sigma_{1}=j$ and $\sigma_{t}=i$ (i.e. the system is in state $j$ at time 0 and in state $i$ at time $t$ ). In turn, the characteristic function of the distribution of $W$ is obtained summing over all the states:

$$
\tilde{f}(t, \lambda)=\sum_{i} \tilde{p}_{i}(t, \lambda)=\sum_{i} \sum_{j}\left[A(\lambda)^{t}\right]_{i j} \nu_{j}(0)=\sum_{j} \nu_{j}(0) \sum_{i}\left[A(\lambda)^{t}\right]_{i j} .
$$

In principle (under suitable analiticity assumptions) one recovers the distribution of $W$ at time $t$ by inverting the transform:

$$
f(t, W)=\int_{-i \infty}^{+i \infty} \mathrm{d} \lambda \exp (\lambda W) \tilde{f}(t, \lambda) .
$$

The knowledge of the characteristic function suffices to generate the moments or the cumulants of $W$ :

$$
\begin{aligned}
\left\langle W_{t}^{n}\right\rangle & =\left.(-1)^{n} \frac{d^{n}}{d \lambda^{n}} \tilde{f}(t, \lambda)\right|_{\lambda=0} \\
\left\langle W_{t}^{n}\right\rangle_{c} & =\left.(-1)^{n} \frac{d^{n}}{d \lambda^{n}} \log \tilde{f}(t, \lambda)\right|_{\lambda=0} .
\end{aligned}
$$


2.2.1. The large time behavior and the $F R_{W}$ At large times, the evolution operator $A(\lambda)^{t}$ is dominated by the largest eigenvalue $y_{1}(\lambda)$ of $A(\lambda)$. Defining $y_{1}(\lambda)=\exp (\zeta(\lambda))$ it follows that

$$
\begin{aligned}
\tilde{\mathbf{p}}(t, \lambda) & \sim \exp (\zeta(\lambda) t)\left[\sum_{j} x_{j}^{(1)}(\lambda) \nu_{j}(0)\right] \mathbf{x}^{(1)}(\lambda) \\
\tilde{f}(t, \lambda)=\sum_{i} \tilde{p}_{i}(t, \lambda) & \sim \exp (\zeta(\lambda) t)\left[\sum_{j} x_{j}^{(1)}(\lambda) \nu_{j}(0)\right] \sum_{i} x_{i}^{(1)}(\lambda),
\end{aligned}
$$

where $\mathbf{x}^{(1)}(\lambda)$ is the eigenvector of $A(\lambda)$ associated to the largest eigenvalue $y_{1}(\lambda)$. One expects, from the above large times behavior, an analogous large time behavior for the density distribution of $W$, i.e.

$$
\begin{aligned}
& f(t, W)=\int_{-i \infty}^{+i \infty} \mathrm{d} \lambda \exp (\lambda W) \sum_{j} \nu_{j}(0) \sum_{i}\left[A(\lambda)^{t}\right]_{i j} \\
& \sim \exp \left[t \max _{\lambda}\left(\lambda \frac{W}{t}+\zeta(\lambda)\right)\right]=\exp [t \pi(W / t)]
\end{aligned}
$$

where we have defined $\pi(w)=\lim _{t \rightarrow \infty} \frac{1}{t} \log f(t, w t)$, the large deviation function associated with $f(t, W)$, which (under the validity of the last chain of equalities) is obtained as a Legendre transform of $\zeta(\lambda)$, i.e. $\pi(w)=\lambda^{*} w+\zeta\left(\lambda^{*}\right)$ with $\left.\frac{d}{d \lambda} \zeta(\lambda)\right|_{\lambda=\lambda^{*}}=$ $-w$.

The $F R_{W}$ symmetry appears at this stage. In fact, it is evident that $A(\lambda)=$ $A^{T}(1-\lambda)$ being $A^{T}$ the transposed of $A$. This implies that $\zeta(\lambda)=\zeta(1-\lambda)$ which suffices to get $\pi(w)=\pi(-w)+w$, i.e. $f(t, W)=f(t,-W) \exp (W)$ at large times.

Clearly the validity of the expansion in (22) is crucial for the validity of the $F R_{W}$ symmetry, but it is not guaranteed when the integrand presents non-analiticities in the $\lambda$ complex plane. Such a catastrophe can happen, for example, when the number of states $N$ becomes infinite and the initial (final) probability $\mu_{j(i)}(0)$ has some unbounded form (see below). The physical meaning of such a catastrophe is that the large fluctuations in the initial and final state cannot be neglected, because they contribute to the tails (i.e. the large deviations) of $f(t, W)$ at any time and, therefore, they prevent the $F R_{W}$ symmetry.

The problem, now, is to find a condition sufficient to trigger the analyticity breaking, in terms of initial/final state distribution. Note that the breaking of analiticity of $\tilde{f}(t, \lambda)$ is associated with large fluctuations of invariant probabilities, but here "large" has a very generic meaning and does not require the presence of any power law tail. Apparently (from many studies including numerical simulations [27], analytical calculations [20, 24] and heuristic arguments, cf. for example [22]), the initial and final configurations can be a problem when the distribution of the boundary term $B=\ln \mu_{\sigma(0)}-\ln \mu_{\sigma(t)}$ has exponential, or higher, tails in the stationary state. But the problem may occur even if $\mu_{i}$ has Gaussian tails! 
2.2.2. The second functional and the finite time symmetry relation Let us see what happens to the "adjusted" functional $W^{\prime}$. Here the prime will always denote quantities which involve this second functional. For example we have the extended measure vector $\mathbf{p}^{\prime}\left(t, W^{\prime}\right)$ and the probability density function at time $t, f^{\prime}\left(t, W^{\prime}\right)$, the characteristic function $\tilde{f}^{\prime}(t, \lambda)$, etc. The increment for the functional at each jump reads $\Delta w_{i j}^{\prime}=$ $\Delta w_{i j} \log \left(\frac{\mu_{i}}{\mu_{j}}\right)$, recalling that $\mu_{i}$ is the invariant measure for the state $i$. The evolution matrix therefore reads

$$
A_{i j}^{\prime}(\lambda)=K_{j i}^{1-\lambda} K_{i j}^{\lambda} \mu_{j}^{-\lambda} \mu_{i}^{\lambda}
$$

and the evolution equation reads

$$
\tilde{\mathbf{p}}^{\prime}(t+1, \lambda)=A^{\prime}(\lambda) \tilde{\mathbf{p}}^{\prime}(t, \lambda) .
$$

The fundamental difference between $W^{\prime}$ and the original $W$ function appears now. Since $\tilde{\mathbf{p}}^{\prime}(0, \lambda)=\boldsymbol{\nu}(0)$, if one takes $\boldsymbol{\nu}(0) \equiv \boldsymbol{\mu}$ (the system is in the stationary regime from the beginning), it happens that

$$
\tilde{f}^{\prime}(1, \lambda)=\sum_{i} \tilde{p}_{i}^{\prime}(1, \lambda)=\sum_{i} \sum_{j} A_{i j}^{\prime}(\lambda) \mu_{j}=\sum_{i} \sum_{j} K_{j i}^{1-\lambda} K_{i j}^{\lambda} \mu_{j}^{1-\lambda} \mu_{i}^{\lambda},
$$

i.e. $\tilde{f}^{\prime}(1, \lambda)=\tilde{f}^{\prime}(1,1-\lambda)$. By recursivity, one realizes that this is the case for all times $t$, i.e.

$$
\tilde{f}^{\prime}(t, \lambda)=\tilde{f}^{\prime}(t, 1-\lambda)
$$

in general, which leads immediately to a finite-time symmetry relation

$$
f^{\prime}\left(t, W^{\prime}\right)=f^{\prime}\left(t,-W^{\prime}\right) \exp \left(W^{\prime}\right)
$$

valid for any $t$.

The second functional contains a term that absorbs all the effects of the fluctuations of the steady state measure, leading to a conservation of the symmetry $\lambda \rightarrow 1-\lambda$ all along the evolution. Such a conservation prevents bad surprises at large times also in presence of large fluctuations of the initial measure.

\subsubsection{Characteristic times When discussing the asymptotic validity of a fluctuation} relation in a finite time simulation an immediate question arises: what are the characteristic times of the evolution and how large with respect to them is the chosen time $\tau$ ? The correct answer, here, is that one is really probing the asymptotic behavior, i.e. really measuring the large deviations of functionals $W$ and $W^{\prime}$, if and only if the fluctuations satisfy the following time scaling:

$$
f(t, W) \sim \exp [t \pi(W / t)] .
$$

Actually, this is equivalent to observe stationary values for the time-dependent cumulants rescaled with the time $c_{n}(t)=\left\langle W(t)^{n}\right\rangle_{c} / t$ and $c_{n}^{\prime}(t)=\left\langle W^{\prime}(t)^{n}\right\rangle_{c} / t$. Verifying the stationarity of the rescaled cumulants sometimes proves to be advantageous with respect to the scaling (28): a cumulant is in fact a simple number containing information on $f(t, W)$ in an integrated form. On the other hand, the stationarity of a cumulant is a 
necessary but not sufficient condition for the validity of the scaling (28) (all cumulants must be stationary) and its measure may eventually be very noisy.

The large time dominance of the maximum eigenvalue allows the direct calculation of the asymptotic cumulants of $W$ and $W^{\prime}$, i.e.

$$
\begin{aligned}
& c_{n}(\infty)=\lim _{t \rightarrow \infty}\left\langle W^{n}\right\rangle_{c} / t=\left.(-1)^{n} \frac{d^{n}}{d \lambda^{n}} \zeta(\lambda)\right|_{\lambda=0} \\
& c_{n}^{\prime}(\infty)=\lim _{t \rightarrow \infty}\left\langle\left(W^{\prime}\right)^{n}\right\rangle_{c} / t=\left.(-1)^{n} \frac{d^{n}}{d \lambda^{n}} \zeta^{\prime}(\lambda)\right|_{\lambda=0} .
\end{aligned}
$$

These formulae are valid provided that $\zeta(\lambda)$ and $\zeta^{\prime}(\lambda)$ are analytic in zero. Numerical observations in the following example suggest a coincidence between $c_{n}(\infty)$ and $c_{n}^{\prime}(\infty)$. This is consistent with direct calculations of $\zeta(\lambda)$ and $\zeta^{\prime}(\lambda)$, which appear to coincide, in Markov chains with a small number of states. We have not been able to address analytically the case of a Markov chain with an infinite number of states. The other evidence which will appear from the simulations (already noticed in the granular tracer model [27]) is that the cumulants of $W$ are much slower than those of $W^{\prime}$ to converge to a stationary value.

The time of convergence to the perfect large deviation scaling (28) is determined in a complex manner by the other eigenvalues (from the second one) of the operator $A(\lambda)$ (or $A^{\prime}(\lambda)$ ). It will be interesting to compare this convergence time (estimated for example from the convergence time of the rescaled cumulants) with the characteristic time of the Markov chain itself $\tau_{M}$. We will use, for $\tau_{M}$, the time obtained with the following standard recipe. An initial vector $\boldsymbol{\nu}(0)$ evolves under the action of the transpose of the transition matrix:

$$
\boldsymbol{\nu}(t)=\left(K^{T}\right)^{t} \boldsymbol{\nu}(0) .
$$

For a generic $\boldsymbol{\nu}(0)$ one has $\boldsymbol{\nu}(t)=\boldsymbol{\mu}+0\left(\mathrm{e}^{-t / \tau_{M}}\right)$ where the characteristic time $\tau_{M}$ is given by the second eigenvalue $\alpha_{2}$ of $K^{T}$, i.e. the closest one to the unitary circle:

$$
\tau_{M}=\frac{1}{\left|\ln \left(\alpha_{2}\right)\right|} .
$$

One can easily compute $\tau_{M}$ from the area $a(t)$ of the parallelogram identified by $\boldsymbol{\mu}$ and $\boldsymbol{\nu}(t)$ :

$$
a(t) \sim \exp \left(-t / \tau_{M}\right)
$$

\section{A Markov chain}

We discuss a simple Markov chain which does not correspond to any particular physical system, but turns out to be useful in illustrating the relevance of the boundary term $B$ in the fluctuations of the two action functionals. Such a model has been inspired by a work by Gaspard and Wang [31]. 


\subsection{The model}

We consider a Markov chain with $N+2$ states, labelled $A, B$ and $C_{i}$ with $i \in\{1,2, \ldots N\}$. The transition matrix $K$ is given by

$$
\left(\begin{array}{llllll}
p_{A A} & p_{A B} & \left(1-p_{A A}-p_{A B}\right) k_{1} & \left(1-p_{A A}-p_{A B}\right) k_{2} & \ldots & \left(1-p_{A A}-p_{A B}\right) k_{N} \\
p_{B A} & 0 & \left(1-p_{B A}\right) k_{1} & \left(1-p_{B A}\right) k_{2} & \ldots & \left(1-p_{B A}\right) k_{N} \\
p_{C A} & 1-p_{C A} & 0 & 0 & \ldots & 0 \\
p_{C A} & 1-p_{C A} & 0 & 0 & \ldots & 0 \\
\vdots & \vdots & & & &
\end{array}\right)
$$

with $\sum_{i=1}^{N} k_{i}=1$, under the constraint that for every jump with nonzero transition probability, the reversed jump is also possible. The invariant probability $\boldsymbol{\mu}$ for $N=1$ is given by

$$
\begin{aligned}
\mu_{A} & =\frac{p_{B A}+p_{C A}-p_{B A} p_{C A}}{\mathcal{N}} \\
\mu_{B} & =\frac{1-p_{A A}+p_{C A}\left(p_{A B}+p_{A A}-1\right)}{\mathcal{N}} \\
\mu_{C} & =\frac{1-p_{A A}-p_{A B} p_{B A}}{\mathcal{N}}
\end{aligned}
$$

with $\mathcal{N}=2+p_{A A}\left(-2+p_{C A}\right)+p_{A B} p_{C A}-p_{B A}\left(-1+p_{A B}+p_{C A}\right)$.

It can be easily seen that the invariant measure for $N>1$ is the same as in the case $N=1$ with a decomposition of the measure of state $C_{1}$ into the measures of $C_{i}$ proportional to the values $k_{i}$ :

$$
\mu_{C_{i}}=\mu_{C} k_{i}
$$

\subsection{Numerical results}

3.2.1. Fluctuations of the functionals We measure the functionals $W(\tau)$ and $W^{\prime}(\tau)$ along independent non-overlapping segments of time-length $\tau$ extracted from a unique trajectory after the stationary regime has been achieved. In particular we probe the validity of a relation like

$$
G_{\tau}(X)=\log F(\tau, X)-\log F(\tau,-X)=X
$$

where $F(t, X)$ is the probability density function of finding one of the two functionals $W$ or $W^{\prime}$ after a time $t$ equal to $X$ (i.e. $F(t, X)$ corresponds to $f(t, W)$ or to $f^{\prime}\left(t, W^{\prime}\right)$ depending on the cases). All the results are shown in figures 1 and 2 containing the graph of $G_{\tau}$ vs $x$ and the pdfs of $W$ and $W^{\prime}$, with different choices of the transition probabilities and of the time $\tau$. We separately discuss three main cases of interest.

1) Whenever $A$ is disconnected from all $C_{i}$ 's, i.e. when $p_{A B}=1-p_{A A}$ and $p_{C A}=0$, detailed balance is satisfied. The numerical results confirm what is expected: in the detailed balance (equilibrium) case, $W^{\prime}(\tau)$ is identically zero and does not fluctuate (its pdf is a delta in zero) and therefore $W(\tau)$ coincides with the opposite of the boundary 
term $B=\log \frac{\mu_{\sigma_{1}}}{\mu_{\sigma_{\tau}}}$ : they both have symmetric fluctuations around zero with exponential tails, and $G_{\tau} \equiv 0$.

2) Lines 1-3 of figure 2. Whenever $A$ is connected to all $C_{i}$ 's, i.e. when $p_{A B}<1-p_{A A}$ and $p_{C A}>0$, detailed balance is violated. In this case both $W(\tau)$ and $W^{\prime}(\tau)$ fluctuate around a nonzero (positive) value which, for $\tau$ large enough, is the same for the two functions. We have chosen $N=50, p_{A A}=0.2, p_{A B}=0.3, p_{B A}=0.3, p_{C A}=0.5$, $\tau=100$. In the first line of graphs of figure 2, we have $k_{i} \propto \exp \left(-i^{2} / 10\right)$, while in all the other frames $k_{i} \propto \exp (-\alpha i)$ with different $\alpha$. The choice of the transition rates $k_{i}$ from state $B$ to state $C_{i}$ determines the invariant measure of states $C_{i}$, which is still proportional to $k_{i}$. Note however that, even in the Gaussian case, the fluctuations of the boundary term $B=\log \frac{\mu_{\sigma_{1}}}{\mu_{\sigma_{\tau}}}$ have a pdf with exponential tails, as confirmed by the numerical observation. The other relevant observation, here, is that the pdf of $W^{\prime}$ is almost perfectly Gaussian. This is not true for the pdf of $W$. At small times $(\tau=100)$ the pdf of $W$ resembles the pdf of $B$, evidencing that at this time $W$ is completely dominated by $B$. At larger times the pdf of $W$ and $B$ start to deviate, in particular that of $W$ tends to become equal to that of $W^{\prime}$, still with evidently different tails of exponential form. The tails of the pdf of $W^{\prime}$ seem to be always dominated by the fluctuations of $B$. Note that (in the third and fourth line of the figure) when the invariant measure is peaked on the first states (i.e. when $\alpha$ is high) the fluctuations of $B$ have the form of a sum of peaks and the pdf of $W=-B+W^{\prime}$ has the form of a sum of Gaussians centered on those peaks.

The symmetry relation for the functional $W$ is not verified in any of these simulations, both at small and large times. The asymmetry measure $G_{\tau}(W)$ has a slope near 1 only for small values of $W \ll\langle W\rangle$, then deviates and saturates to a constant value in good agreement with the value $2\langle W\rangle$ predicted by van Zon and Cohen [22], also in agreement with ref. [26]. Note that the observations at large times are perfectly compatible with those at small times, i.e. if both ordinates and abscisass are divided by $\tau$ the curves are similar (in the first two cases they collapse very well). Therefore the "reduction" of the violation at large times is only apparent. On the other hand the symmetry relation for $W^{\prime}$ is always satisfied, at all times and for all the choices of the parameters.

3) Fourth line of figure 2. In this simulation the invariant measure on states $C_{i}$ is still an exponential, but with a higher slope. In this case our numerical results show that the fluctuations of $W$ are much closer to those of $W^{\prime}$ already at small times. This is reflected on the good agreement with the Fluctuation Relation of both functionals $W$ and $W^{\prime}$. Note that one can still be doubtful about this verification, because of the limited range of values of $W$ available, which can possibly hide a failure at larger values. The distribution of the boundary term in fact still has exponential tails (invisible at our resolution) and again it can be argued that these tails (being Gaussian those of $W^{\prime}$ ) will dominate at very large values. 

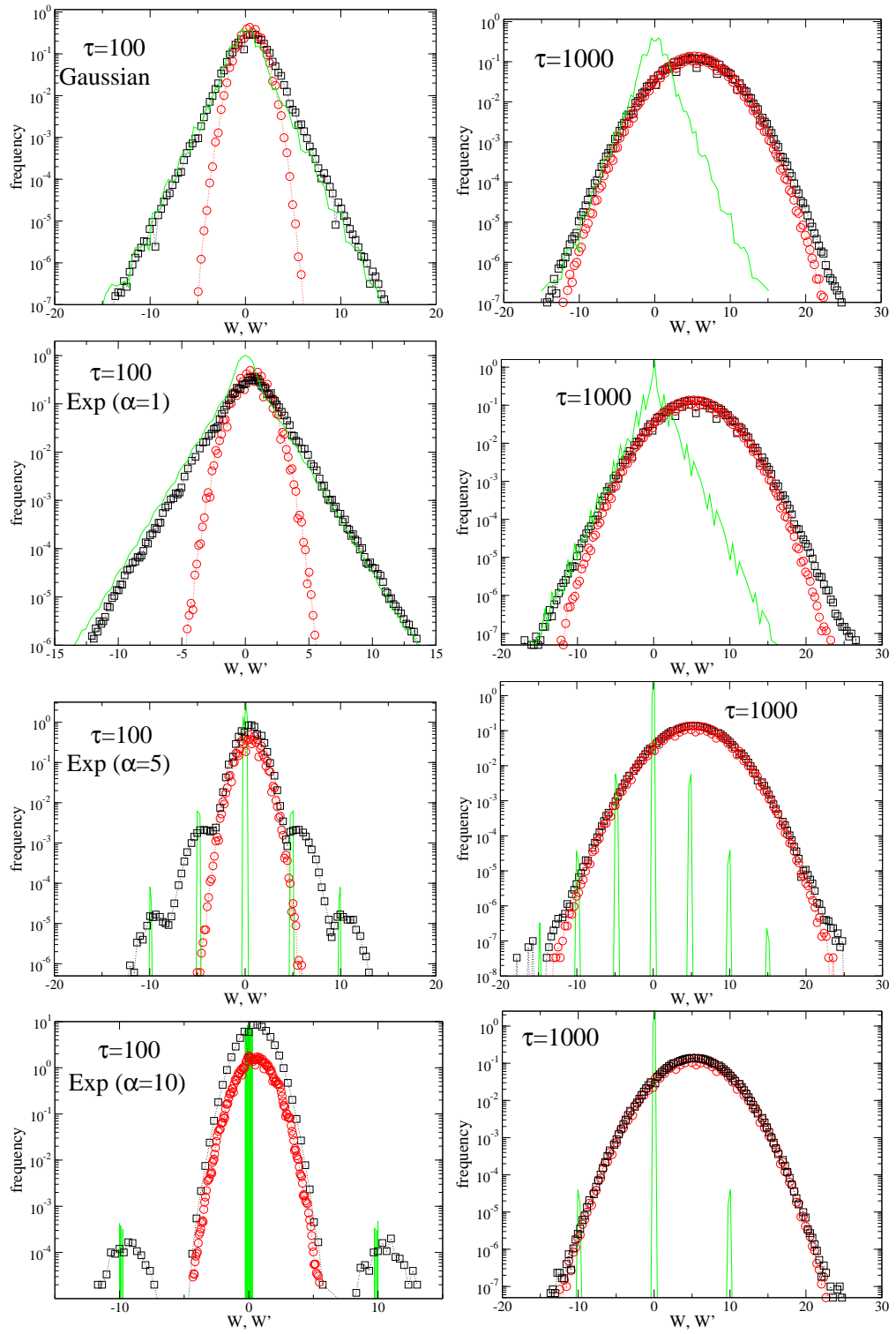

Figure 1. Color online. Probabilities of observing $W$ (black squares), $W^{\prime}$ (red circles) and $B=W^{\prime}-W$ (green lines), for the Markov chain. Each line is composed of two graphs and shows the results for a particular choice of the parameters: the left column is at time $\tau=100$ and the right column at time $\tau=1000$. In all the simulations we have used $N=50$. In all frames we have used $p_{A A}=0.2$, $p_{A B}=0.3, p_{B A}=0.3$ and $p_{C A}=0.5$ : the first line corresponds to Gaussian tails of the invariant measure, because $k_{i} \propto \exp \left(-i^{2} / 10\right)$, while the last three lines correspond to exponential choices $k_{i} \propto \exp (-\alpha i)$ with the value of $\alpha$ given in the plot. In all cases $\langle W(\tau)\rangle=\left\langle W^{\prime}(\tau)\right\rangle=0.0053 \tau$, i.e. 0.53 for $\tau=100$ and 5.3 for $\tau=1000$. 

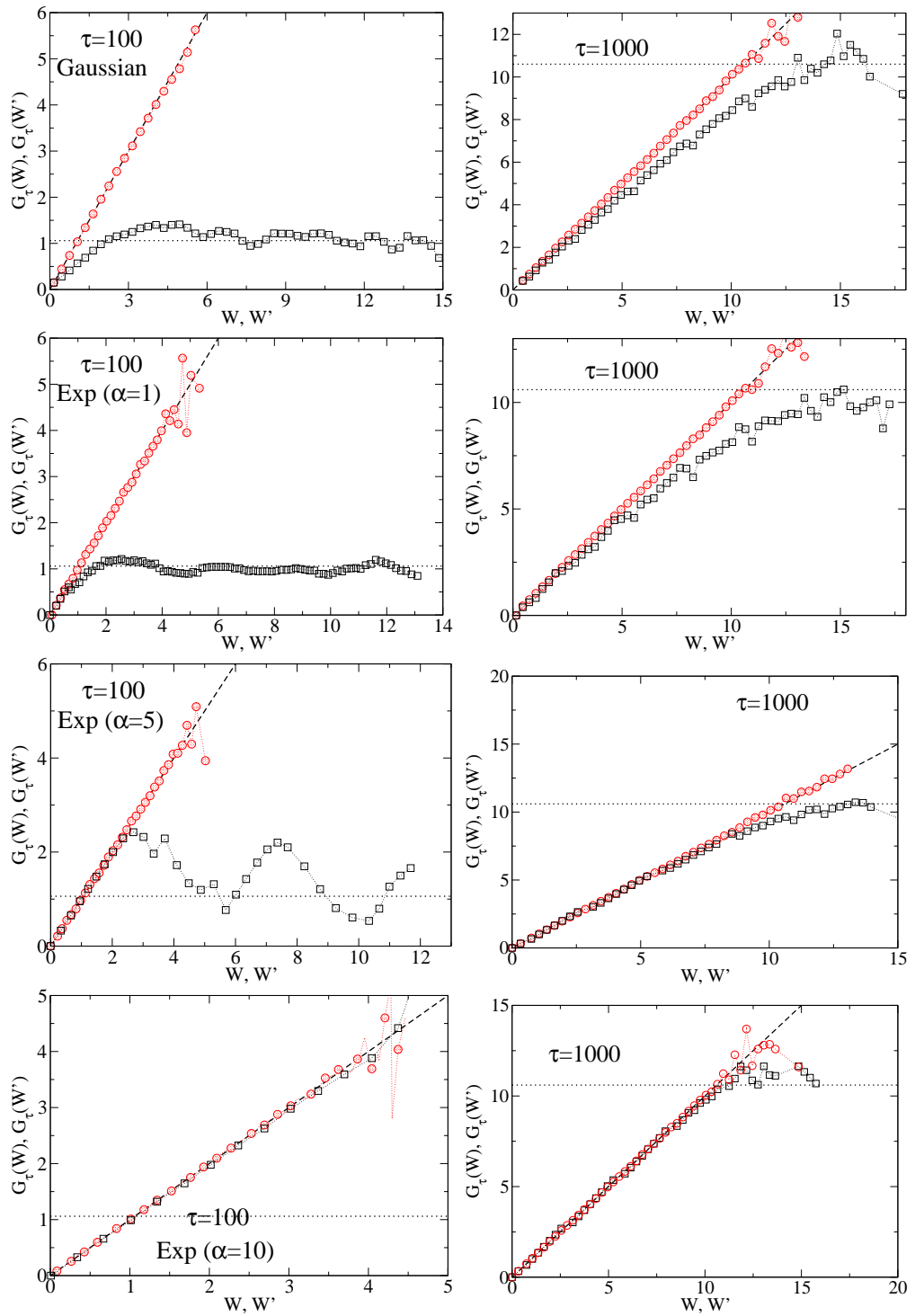

Figure 2. Color online. $G_{\tau}(W)$ vs. $W$ (black squares) and $G_{\tau}\left(W^{\prime}\right)$ vs. $W^{\prime}$ (red circles) for the Markov chain. Each line is composed of two graphs and shows the results for a particular choice of the parameters: the left column is at time $\tau=100$ and the right column at time $\tau=1000$. The dashed line has slope 1 . In all the simulations we have used $N=50$. In all frames we have used $p_{A A}=0.2$, $p_{A B}=0.3, p_{B A}=0.3$ and $p_{C A}=0.5$ : the first line corresponds to Gaussian tails of the invariant measure, because $k_{i} \propto \exp \left(-i^{2} / 10\right)$, while the last three lines correspond to exponential choices $k_{i} \propto \exp (-\alpha i)$ with the value of $\alpha$ given in the plot. In all cases $\langle W(\tau)\rangle=\left\langle W^{\prime}(\tau)\right\rangle=0.0053 \tau$, i.e. 0.53 for $\tau=100$ and 5.3 for $\tau=1000$. The dotted horizontal line marks the van Zon and Cohen prediction for the FR violation, $G_{\tau}(W)=2\langle W\rangle$ for large $W[22$. 
3.2.2. Characteristic times: scaling and cumulants Following the recipe given in section 2.2.3, we have calculated the characteristic time $\tau_{M}$ associated with the approach toward the invariant probability of the Markov chain. Our general observation is that its dependence upon the choice of the particular form of the transition probabilities $k_{i}$, which couple the state $B$ with the $N$ states $C_{i}$, is negligible. In particular, for all the choice of parameters corresponding to the cases shown in figure 2, we have measured $\tau_{M} \approx 2.1$, while in the equilibrium case $p_{A A}=0.2, p_{A B}=0.8, p_{B A}=0.3$ and $p_{C A}=0$, we have measured $\tau_{M} \approx 18$.

In figure 3-left we plot the large deviation rate function at finite times $\Pi(t, x)$ for the two functionals $W$ and $W^{\prime}$, in a non-equilibrium case with a choice of $k_{i}$ such that the fluctuations of the invariant measure are large, i.e. $k_{i} \propto \exp (-i)$ (the $\alpha=1$ case discussed in the previous section). The range of available values drops very rapidly as $t$ increases, and, most importantly, the negative range tends rapidly to disappear. On the basis of these results one can conclude that the scaling (28) is already reached at $t=100$. This is true for both functionals, $W$ and $W^{\prime}$. The two large deviation rate functions appear different for $W$ and $W^{\prime}$, they seem to have linear and quadratic tails respectively.

In figure 3 -right we show the values of the first four cumulants (divided by the time, i.e. $c_{n}(t)$ and $c_{n}^{\prime}(t)$ as defined previously) of $W(t)$ and $W^{\prime}(t)$, as a function of time $t$. The absolute values have been taken, in order to use a logarithmic plot and better appreciate the different orders of magnitude. In each frame of the figure the value of $c_{n}^{\prime}(1000)$ is given. One first observes that the cumulants of $W^{\prime}$ become time-independent almost immediately. The values of the third and fourth cumulant are consistent with the previous observation that the fluctuations of $W^{\prime}$ are almost Gaussian, and its large deviation rate function is quadratic. The remarkable fact is that the cumulants of $W$ are much slower to converge to a stationary value: as a matter of fact we cannot conclude, in our case, that this convergence has been actually reached apart from the first cumulant. The measure of the cumulants points out the weakness of a naked-eye verification of the large deviation scaling as the one obtained in figure 3-left.

\section{The granular tracer}

Recently, both in experiments [32] and in simulations [33], the validity of the $F R_{W}$ has been studied in granular gases. A granular gas consists of inelastic particles, typically one has a box containing macroscopic grains (like sand) strongly vibrated in order to obtain a dilute and fluidized stationary regime, which is far from equilibrium because of nonconservative forces acting on and among the grains. In a series of other papers [34] it has been shown that the prototypical model of granular gas, the gas of inelastic hard spheres, cannot be used without some care as a benchmark for the validity of the fluctuation relation: each single collision violates time reversibility, so that for any trajectory in the phase space of the whole system, its reversed trajectory does not exist. This implies that the large deviations of entropic functionals are defined only for positive valuess, and 

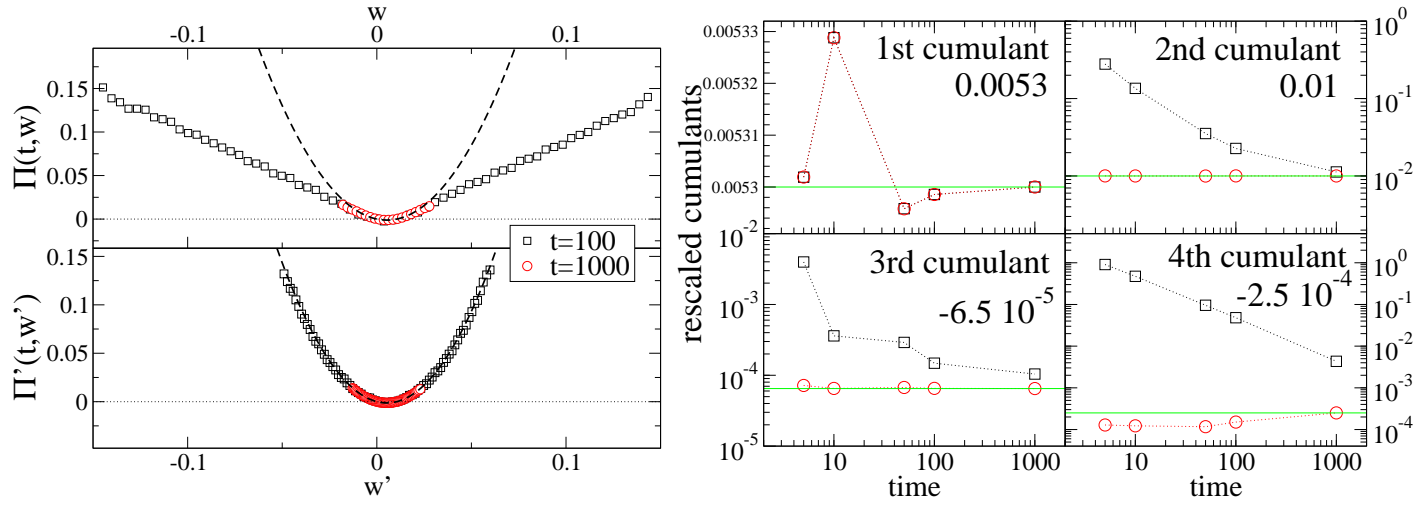

Figure 3. Color online. Left: the large deviation function rate at finite times $\Pi(\tau, x)-\Pi(\tau, 0)=-\frac{1}{\tau} \log \frac{F(\tau, x)}{F(\tau, 0)}$ for the fluctuations of $W / \tau$ (top frame) and of $W^{\prime} / \tau$ (bottom frame). The dashed line shows the quadratic function associated with the Gaussian of same mean and same asymptotic variance, while the dotted line marks 0 . Right: the first four time-rescaled cumulants of $W$ (squares) and $W^{\prime}$ (circles) as a function of time. The solid green line represents the absolute value of the cumulant of $W^{\prime} / \tau$ at $\tau=1000$ : this value is also indicated in each frame. The parameters are the same as in figure 1 second line (i.e. $k_{i} \propto \exp -i$ ).

that the fluctuation relation cannot be verified. However, focussing on the Markovian dynamics of a single tracer particle immersed in a gas, one may circumvent the problem of strong irreversibility and probe the validity of the FR in granular gases [27.

\subsection{The model}

We consider the dynamics of a tracer granular particle in a homogeneous and dilute gas of grains which is driven by an unspecified energy source. The tracer particle experiences consecutive collisions with particles of the gas coming from the same "population", independently of the position and time of collision. The gas is characterized by its velocity probability density function $P(\mathbf{v})$, which is well known to be mildly nonGaussian and well reproduced by a first Sonine correction 35. The dilution of the gas guarantees that the transient as well as the stationary regimes of the velocity probability density function $P_{*}(\mathbf{v})$ of the tracer is governed by a Linear Boltzmann equation.

The inelastic collisions with the gas particles, which determine the instantaneous changes of the velocity of the tracer, are described by the simplest and most used inelastic collision rule:

$$
\mathbf{v}^{\prime}=\mathbf{v}-m \frac{1+\alpha}{m+M}[(\mathbf{v}-\mathbf{V}) \cdot \hat{\boldsymbol{\omega}}] \hat{\boldsymbol{\omega}}
$$

where $\mathbf{v}$ and $\mathbf{v}^{\prime}$ are the velocities of the tracer before and after the collision respectively, $\mathrm{V}$ is the velocity of the gas particle, $m$ and $M$ are the masses of the tracer and of the gas particle respectively, $\hat{\boldsymbol{\omega}}$ is the unitary vector joining the centers of the two particles, and $\alpha$ is the restitution coefficient that takes values in $[0,1]$ ( 1 is the elastic case). For simplicity, in the rest of the discussion we will consider 2-dimensional systems with $m=M$ (see [27] for a more general discussion). 
The analysis of the Linear Boltzmann equation leads to the following Master equation for the evolution of the velocity probability density function of the tracer:

$$
\frac{\mathrm{d} P_{*}(\mathbf{v}, t)}{\mathrm{d} t}=\int \mathrm{d} \mathbf{v}_{1}\left[P_{*}\left(\mathbf{v}_{1}\right) K\left(\mathbf{v}_{1}, \mathbf{v}\right)-P_{*}(\mathbf{v}) K\left(\mathbf{v}, \mathbf{v}_{1}\right)\right] .
$$

where $P_{*}(v)$ is the velocity pdf of the test particle. The transition rate $K\left(\mathbf{v}, \mathbf{v}^{\prime}\right)$ (see [27] for a precise definition) of jumping from $\mathbf{v}$ to $\mathbf{v}^{\prime}$ is given by the following formula:

$$
K\left(\mathbf{v}, \mathbf{v}^{\prime}\right)=\left(\frac{2}{1+\alpha}\right)^{2} \int \mathrm{d} v_{2 \tau} P\left[\mathbf{v}_{2}\left(\mathbf{v}, \mathbf{v}^{\prime}, v_{2 \tau}\right)\right],
$$

where $\Delta \mathbf{v}=\mathbf{v}^{\prime}-\mathbf{v}$ denotes the change of velocity of the test particle after a collision and $P(\mathbf{v})$ is the velocity pdf of the gas. In Eq. (42) it has been assumed that the mean free path equals 1 , which can be always obtained rescaling the time. This means that the elastic mean free time is $\tau_{c}^{e l}=1 / 2 \sqrt{\pi}$ (it is larger in inelastic cases). The vectorial function $\mathbf{v}_{2}$ is defined as

$$
\mathbf{v}_{2}\left(\mathbf{v}, \mathbf{v}^{\prime}, v_{2 \tau}\right)=v_{2 \sigma}\left(\mathbf{v}, \mathbf{v}^{\prime}\right) \hat{\boldsymbol{\sigma}}\left(\mathbf{v}, \mathbf{v}^{\prime}\right)+v_{2 \tau} \hat{\boldsymbol{\tau}}
$$

where $\hat{\boldsymbol{\sigma}}\left(\mathbf{v}, \mathbf{v}^{\prime}\right)$ is the unitary vector parallel to $\Delta \mathbf{v}$ while $\hat{\boldsymbol{\tau}}$ is the unitary vector perpendicular to it. Finally, to fully determine the transition rate (42), the expression of $v_{2 \sigma}$ is needed:

$$
v_{2 \sigma}\left(\mathbf{v}, \mathbf{v}^{\prime}\right)=\frac{2}{1+\alpha}|\Delta \mathbf{v}|+\mathbf{v} \cdot \hat{\boldsymbol{\sigma}}
$$

For the purpose of measuring the fluctuations of the functionals defined by Eq. (8), Eq. (42) yields:

$$
\frac{K\left(\mathbf{v}, \mathbf{v}^{\prime}\right)}{K\left(\mathbf{v}^{\prime}, \mathbf{v}\right)}=\frac{\int \mathrm{d} v_{2 \tau} P\left[\mathbf{v}_{2}\left(\mathbf{v}, \mathbf{v}^{\prime}, v_{2 \tau}\right)\right]}{\int \mathrm{d} v_{2 \tau} P\left[\mathbf{v}_{2}\left(\mathbf{v}^{\prime}, \mathbf{v}, v_{2 \tau}\right)\right]} \equiv \frac{P\left[v_{2 \sigma}\left(\mathbf{v}, \mathbf{v}^{\prime}\right)\right]}{P\left[v_{2 \sigma}\left(\mathbf{v}^{\prime}, \mathbf{v}\right)\right]}
$$

Then we consider two possible choices for the velocity pdf of the gas, a Gaussian and a non-Gaussian case expressed in the form of a first Sonine correction to the Gaussian [36], which amounts to $P(\mathbf{v})=\frac{1}{(2 \pi T)} \exp \left(-\frac{v^{2}}{2 T}\right)\left(1+a_{2} S_{2}^{2}\left(v^{2} / 2 T\right)\right)$. The first Sonine correction enters by means of the small parameter $a_{2}$ (which is 0 in the Gaussian case) and the Sonine Polynomial $S_{2}^{d}(x)=\frac{1}{2} x^{2}-\frac{d+2}{2} x+\frac{d(d+2)}{8}$, which depends upon the choice of physical parameters, e.g. density, restitution coefficient, etc. The most realistic case is the non-Gaussian one, which is common to all experiments and simulations, while the Gaussian case is presented for reference (after the study of Martin and Piasecki 37]). In the Gaussian case we get

$$
\log \frac{K\left(\mathbf{v}, \mathbf{v}^{\prime}\right)}{K\left(\mathbf{v}^{\prime}, \mathbf{v}\right)}=\frac{\Delta}{2 T}+2 \frac{1-\alpha}{1+\alpha} \frac{\Delta}{2 T}=\frac{3-\alpha}{1+\alpha} \frac{\Delta}{2 T},
$$

with $\Delta=v_{\sigma}^{2}-\left(v_{\sigma}^{\prime}\right)^{2} \equiv|v|^{2}-\left|v^{\prime}\right|^{2}$, i.e. the kinetic energy lost by the test-particle during one collision. In the First Sonine correction case it is instead obtained

$$
\log \frac{K\left(\mathbf{v}, \mathbf{v}^{\prime}\right)}{K\left(\mathbf{v}^{\prime}, \mathbf{v}\right)}=\frac{3-\alpha}{1+\alpha} \frac{\Delta}{2 T}+\log \frac{\left\{1+a_{2} S_{2}^{d=1}\left[\frac{\left(\frac{2}{1+\alpha}\left(v_{\sigma}^{\prime}-v_{\sigma}\right)+v_{\sigma}\right)^{2}}{2 T}\right]\right\}}{\left\{1+a_{2} S_{2}^{d=1}\left[\frac{\left(\frac{2}{1+\alpha}\left(v_{\sigma}-v_{\sigma}^{\prime}\right)+v_{\sigma}^{\prime}\right)^{2}}{2 T}\right]\right\}}
$$


In the case where $P(v)$ is a Gaussian with temperature $T$, it is immediate to observe that

$$
P_{*}(\mathbf{v}) K\left(\mathbf{v}, \mathbf{v}^{\prime}\right)=P_{*}\left(\mathbf{v}^{\prime}\right) K\left(\mathbf{v}^{\prime}, \mathbf{v}\right)
$$

if $P_{*}$ is equal to a Gaussian with temperature $T^{\prime}=\frac{\alpha+1}{3-\alpha} T \leq T$. This means that there is a Gaussian stationary solution of equation (41) (in the Gaussian-bulk case), which satisfies detailed balance. The fact that such a Gaussian with a different temperature $T^{\prime}$ is an exact stationary solution was known from [37]. It thus turns out that detailed balance is satisfied in these cases, even in the absence of thermal equilibrium. Of course this is an artifact of our model: it is indeed highly unrealistic that a granular gas yields a Gaussian velocity pdf. As soon as the gas velocity pdf $P(v)$ ceases to be Gaussian, detailed balance is violated, i.e. the stationary process performed by the tracer particle is no more in equilibrium within the thermostatting gas.

\subsection{Numerical results}

The dynamics of a tracer particle undergoing inelastic collisions with a gas of particles in a stationary state with a given velocity pdf $P(\mathbf{v})$ has been simulated by means of the Direct Simulation Monte Carlo algorithm [38. We have three parameters: the restitution coefficient $\alpha$ of collisions between the tracer and the rest of the gas, the temperature of the gas $T$ (which we take as unity) and the coefficient of the first Sonine correction which parametrizes the velocity pdf of the gas, $a_{2}$. The tracer particle has a measured "temperature" (mean kinetic energy) $T_{*}<T$ and a velocity pdf that is observed to be well described again by a first Sonine correction to a Gaussian, parametrized by a coefficient $a_{2}^{*}$. To measure the quantity $W^{\prime}(\tau)=W(\tau)+B$ on each segment of trajectory we assume this observation to be exact and we use the values $T_{*}$ and $a_{2}^{*}$ measured during the simulation itself to compute the "boundary term" $B$. All the results are shown in figures 4 and 5. for two different choices of the time $\tau$ and many choices of the parameters $\alpha$ and $a_{2}$. We display in each figure the value of $\langle w\rangle=\lim _{\tau \rightarrow \infty} W(\tau) / \tau$. Again the idea that this quantity measures the distance from equilibrium of our system is well supported by the results of the simulations: $\langle w\rangle$ is zero when $a_{2}=0$ and increases as $\alpha$ is decreased and $a_{2}$ is increased. We recall that $\lim _{\tau \rightarrow \infty} W(\tau) / \tau=\lim _{\tau \rightarrow \infty} W^{\prime}(\tau) / \tau \equiv\langle w\rangle$, since the difference between the two functionals has zero average at large times $\tau$. We also remark that the distribution of both quantities $W$ and $W^{\prime}$ are symmetric at equilibrium (i.e. when $a_{2}=0$ ).

We first discuss the results concerning the fluctuations of $W(\tau)$, identified in figures 4 and 5 by squared symbols. They are strongly non-Gaussian, with almost exponential tails, at low values of $\tau$ for any choice of the parameters. At large values of $\tau$ (many hundreds of mean free times), the situation changes with how far from equilibrium the system is. At low values of $\langle w\rangle$ the tails of the distribution are very similar to the ones observed at small times. At higher values of $\langle w\rangle$ the distribution changes with time and tends to become more and more Gaussian. We have not been able to measure negative deviations larger than the ones shown in the figures. Distributions 
obtained at higher values of $\tau$ yielded a smaller statistics and very few negative events. With these numerical observations, we could not verify the $F R_{W}$ relation. In the last case, which is very far from equilibrium $\left(a_{2}=0.3, \alpha=0\right)$, a behavior compatible with $F R_{W}$ is observed, i.e. $G_{\tau}(W) \approx W$, but the range of available values of $W$ is much less than $\langle W\rangle$. At this stage, and in practice, we consider such results a failure of the $F R_{W}$ for continuous Markov processes. Our numerical results are also in fair agreement with the prediction by van Zon and Cohen for the violation of the $F R_{W}$, i.e. (at least for large times and not too far from equilibrium) $G_{\tau}(W) \sim 2\langle W\rangle$. The deviations from the Gaussian of the pdfs of $W^{\prime}$ are weaker than those observed for $W$, and the $F R_{W^{\prime}}$ is well satisfied in all non-equilibrium cases at all times $\tau$. The verification of both FRs is either trivial or meaningless in the equilibrium cases $\left(a_{2}=0\right)$, where $G_{\tau} \equiv 0$.

\section{Conclusions}

We conclude this paper with the following observations. In the first place, it seems important to point out the remarkable similarity of the results obtained for a rather abstract Markov chain and the granular tracer. Because the observables considered in this paper are subtle, one may expect that the similarity between the abstract and the concrete models concerns other observables as well, which is worth further investigation.

We have observed a failure of the fluctuation relation for $W\left(F R_{W}\right)$, at all times that we could consider. This does not exclude that the relation holds at much larger times, which are of no practical interest, although they can be of theoretical interest. The failure is due to the boundary terms, which become more and more important as the steady state draws closer and closer to equilibrium. This suggests that the difference between physically relevant times, and the times at which the $F R_{W}$ might be verified, grows as the steady state approaches an equilibrium state, possibly diverging in the equilibrium limit.

The (practical) failure of the $F R_{W}$ reported here does agree in a quantitative fashion with the one predicted by Van Zon and Cohen, which was thought to be at work also in some deterministic systems [26]. However, a different conclusion is afforded by the results of 29, which indicates that the possible failures of the $F R_{W}$ do not have a universal character, and that further investigations are desirable.

The general conclusion that we can draw from the above observations is that large deviations are particularly subtle objects, whose peculiarities are not so obvious in the physics of equilibrium systems, but become evident as soon as a slight perturbation away from equilibrium sets in. This should not sound paradoxical: steady states are achieved asymptotically in time; this allows phenomena, such as long range correlations, which are extraneous to the physics of most equilibrium systems, but are well known in the physics of nonequilibrium steady states. Furthemore, to keep a system on a nonequilibrium steady state, an unlimited amount of work may be done on the system or extracted from it, and this is bound to have observable (and perhaps exploitable) consequences on the behaviour of the system. 

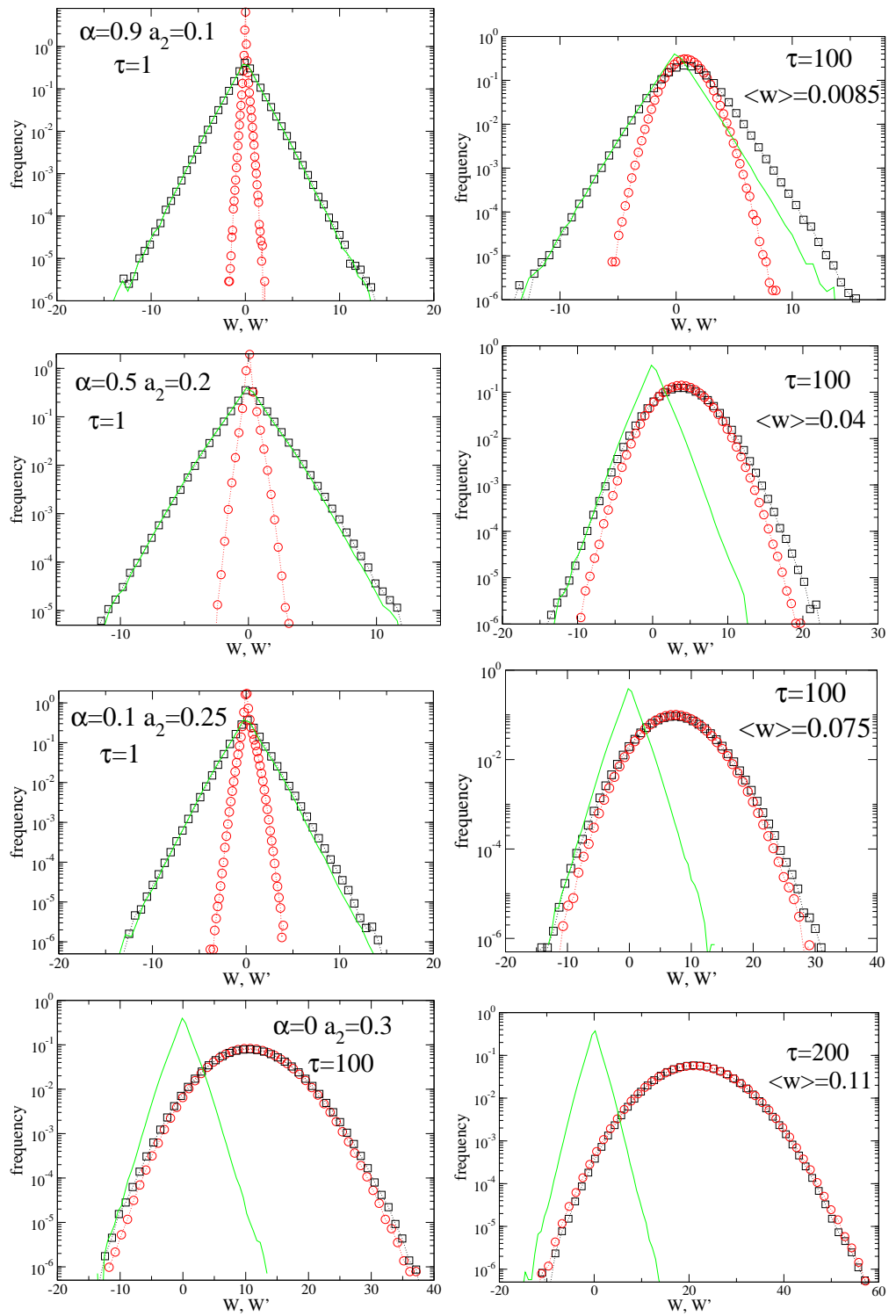

Figure 4. Color online. Probability of fluctuations of $W(\tau)$ (black squares), $W^{\prime}(\tau)$ (red circles) and $B=W^{\prime}-W$ (green line) for the continuous Markov process defined by the dynamics of a tracer in a granular gas. Each line is composed of two graphs and shows the results for a particular choice of $\alpha$ and $a_{2}$ (the coefficient of the first Sonine correction characterizing the non-Gaussianity of the velocity pdf of the gas): the left graphs are at small times, while the right graphs are at large times. Mean free time between collisions slightly varies with parameters, but is always near $\tau_{c} \sim 0.3$. For each choice of parameters, inside the right graph, it is shown the value of $\langle W(\tau)\rangle$ and of $\langle w\rangle \equiv\langle W(\tau)\rangle / \tau \equiv\left\langle W^{\prime}(\tau)\right\rangle / \tau \equiv \lim _{\tau \rightarrow \infty} W(\tau) / \tau \equiv \lim _{\tau \rightarrow \infty} W^{\prime}(\tau) / \tau$ which can be considered a distance from equilibrium. 

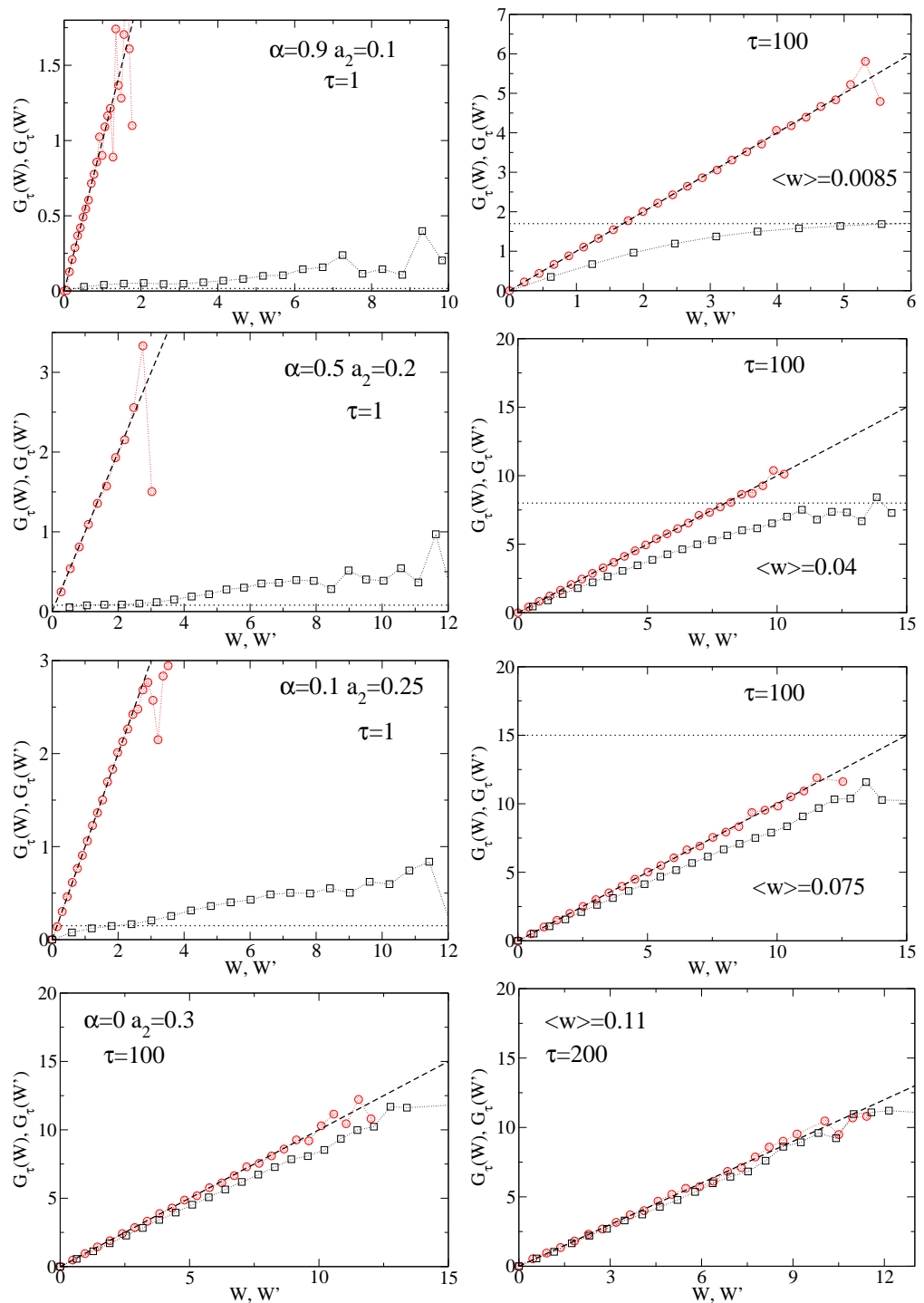

Figure 5. Color online. $G_{\tau}(W)$ vs. $W$ (black squares) and $G_{\tau}\left(W^{\prime}\right)$ vs. $W^{\prime}$ (red circles) for the continuous Markov process defined by the dynamics of a tracer in a granular gas. Each line is composed of two graphs and shows the results for a particular choice of $\alpha$ and $a_{2}$ (the coefficient of the first Sonine correction characterizing the nonGaussianity of the velocity pdf of the gas): the left graphs is at small times and the right graphs at large times. For each choice of parameters, inside the right graph, it is shown the value of $\langle W(\tau)\rangle$ and of $\langle w\rangle \equiv\langle W(\tau)\rangle / \tau \equiv\left\langle W^{\prime}(\tau)\right\rangle / \tau \equiv \lim _{\tau \rightarrow \infty} W(\tau) / \tau \equiv$ $\lim _{\tau \rightarrow \infty} W^{\prime}(\tau) / \tau$ which can be considered a distance from equilibrium. The dashed line has slope 1. The dotted line represents the van Zon and Cohen prediction for the $F R_{W}$ violation, $G_{\tau}(W)=2\langle W\rangle$ for large $W[22$. It is not visible in the fourth line. 
Acknowledgments.- A. P. acknowledges the support of the EC grant MERG-021847. The authors are indebted to D. J. Searles for discussions, hospitality and a critical reading of the manuscript. A. P. also acknowledges discussions with G. Gallavotti, P. Visco, F. van Wijland, and F. Zamponi.

\section{References}

[1] Zwanzig R, Nonequilibrium Statistical Mechanics, 2001 Oxford University Press Inc, USA.

[2] Gallavotti G, Statistical Mechanics: A Short Treatise, 1999 Springer-Verlag, Berlin.

[3] Kreuzer HJ, Non-equilibrium Thermodynamics and Its Statistical Foundations, 1983 Oxford University Press.

[4] Einstein A, Ueber die von der molekularkinetischen Theorie der Waerme geforderte Bewegung von in ruhenden Fluessigkeiten suspendierten Teilchen, 1905 Ann. d. Phys. 17, 549.

[5] Onsager L, Reciprocal Relations in Irreversible Processes, 1931 Phys. Rev. 37, 405; 1931 Phys. Rev. 38, 2265.

[6] Green MS, Markoff Random Processes and the Statistical Mechanics of Time-Dependent Phenomena. II. Irreversible Processes in Fluids, 1954 J. Chem. Phys. 22, 398.

[7] Statistical-Mechanical Theory of Irreversible Processes. I. General Theory and Simple Applications to Magnetic and Conduction Problems, Kubo R, 1957 J. Phys. Soc. Japan 12, 570.

[8] de Groot SR and Mazur P, 1969 Non-equilibrium thermodynamics, North-Holland.

[9] Rose RH and Sulem PL, Fully developed turbulence and statistical mechanics, 1978 J. Phys (Paris) 39, 441.

[10] Falcioni M, Isola S and Vulpiani A, Correlation functions and relaxation properties in chaotic dynamics and statistical mechanics, 1990 Phys. Lett. A 144, 341.

[11] Falcioni M and Vulpiani A, The relevance of chaos for the linear response theory, 1995 Physica A. 215, 481.

[12] Boffetta G, Lacorata G, Musacchio S and Vulpiani A, Relaxation of finite perturbations: Beyond the Fluctuation-Response relation, 2003 Chaos 13, 806.

[13] Evans DJ, Cohen EGD and Morriss GP, Probability of second law violations in shearing steady states, 1993 Phys. Rev. Lett. 71, 2401.

[14] Evans DJ and Searles DJ, Equilibrium microstates which generate second law violating steady states, 1994 Phys. Rev. E 50, 1645.

[15] Gallavotti G and Cohen EGD, Dynamical Ensembles in Nonequilibrium Statistical Mechanics, 1995 Phys. Rev. Lett. 74, 2694.

[16] Lebowitz JL and Spohn H, A Gallavotti-Cohen-Type Symmetry in the Large Deviation Functional for Stochastic Dynamics, 1999 J. Stat. Phys. 95, 333.

[17] Maes C, The Fluctuation Theorem as a Gibbs Property, 1999 J. Stat. Phys. 95, 367.

[18] Kurchan J, Fluctuation theorem for stochastic dynamics, 1998 J. Phys. A.: Math. Gen. 31, 3719.

[19] Jarzynski C, Nonequilibrium Equality for Free Energy Differences, 1997 Phys. Rev. Lett. 78, 2690; Crooks GE, 1999 Phys. Rev. E 60, 002721.

[20] Farago J, Injected Power Fluctuations in Langevin Equation, 2002 J. Stat. Phys. 107, 781; Power fluctuations in stochastic models of dissipative systems 2004 Physica A 331, 69.

[21] Evans DJ and Searles DJ, The fluctuation theorem, 2002 Adv. Phys. 51, 1529.

[22] van Zon R and Cohen EGD, Extension of the Fluctuation Theorem, 2003 Phys. Rev. Lett. 91, 110601.

[23] Baiesi M, Jacobs T, Maes C and Skantzos NS, Fluctuation symmetries for work and heat. cond-mat/0602311

[24] Visco P, Work fluctations for a Brownian particle between two thermostats, 2006 J. Stat. Mech. P06006. 
[25] Dolowschiak M, Kovacs Z, Fluctuation formula and non-Gaussian distribution of the entropy production rate in the thermostated Lorentz gas, 2005 Phys. Rev. E 71, 025202(R).

[26] Bonetto F, Gallavotti G, Giuliani A and Zamponi F, Chaotic Hypothesis, Fluctuation Theorem and singularities, 2006 J. Stat. Phys. 123, 39.

[27] Puglisi A, Visco P, Trizac E and van Wijland F, Dynamics of a tracer granular particle as a nonequilibrium Markov process, 2006 Phys. Rev. E. 73, 021301.

[28] Seifert U, Entropy Production along a Stochastic Trajectory and an Integral Fluctuation Theorem, 2005 Phys. Rev. Lett. 95, 040602.

[29] Harris RJ, Rákos A and Schütz M, Breakdown of Gallavotti-Cohen symmetry for stochastic dynamics, cond-mat/0512159.

[30] Gaspard P, Time-Reversed Dynamical Entropy and Irreversibility in Markovian Random Processes, 2004 J. Stat. Phys. 117, 599.

[31] Gaspard P and Wang X-J, Sporadicity: Between Periodic and Chaotic Dynamical Behaviors, 1998 Proc. Natl. Acad. Sci. USA 83, 4591.

[32] Feitosa K and Menon N, Fluidized Granular Medium as a an Istance of the Fluctuation Theorem, 2004 Phys. Rev. Lett. 92, 164301.

[33] Aumaitre S, Fauve S, McNamara S and Poggi P, Power injected in dissipative systems and the fluctuation theorem, 2001 Eur. Phys. J. B 19, 449.

[34] Visco P, Puglisi A, Barrat A, Trizac E and van Wijland F, Injected power and entropy flow in a heated granular gas, 2005 Europhys. Lett. 72, 55; Puglisi A, Visco P, Barrat A, Trizac E and van Wijland F, Fluctuations of Internal Energy Flow in a Vibrated Granular Gas, 2005 Phys. Rev. Lett. 95, 110202; Visco P, Puglisi A, Barrat A, Trizac E and van Wijland F, 2006 J. Stat. Phys. in press.

[35] Williams DRM and MacKintosh FC, Driven granular media in one dimension: Correlations and equation of state, 1996 Phys. Rev. E 54 R9; Puglisi A, Loreto V, Marconi UMB, Petri A and Vulpiani A, Clustering and non-Gaussian Behavior in Granular Matter, 1998 Phys. Rev. Lett. 81, 3848; van Noije TPC and Ernst M, Velocity Distributions in Homogeneously Cooling and Heated Granular Fluids, 1998 Gran. Matter 1, 57; van Noije TPC, Ernst MH, Trizac E and Pagonabarraga I, Randomly driven granular fluids: Large-scale structure, 1999 Phys. Rev E 59, 4326; Henrique C, Batrouni G and Bideau D, Diffusion as a mixing mechanism in granular materials, 2000 Phys. Rev. E 63, 011304; Pagonabarraga I, Trizac E, van Noije TPC and Ernst MH, Randomly driven granular fluids: Collisional statistics and short scale structure, 2002 Phys. Rev. E 65, 011303.

[36] Sonine polynomials are often used to construct solutions to the Boltzmann equation. They are exact eigenfunctions of the linearized Boltzmann collision operator for elastic Maxwell molecules, i.e. particles interacting with repulsive $r^{-4}$ potentials. They are discussed in great detail in S. Chapman and T. G. Cowling, The Mathematical Theory of Non-Uniform Gases, Cambridge University Press, 1970.

[37] Martin PA and Piasecki J, Thermalization of a particle by dissipative collisions, 1999 Europhys. Lett. 46, 613.

[38] Bird GA, Molecular Gas Dynamics and the Direct Simulation of Gas Flows, 1994 Clarendon 1994 (Oxford); Montanero MJ and Santos A, 2000 Granular Matter 2, 53. 